Acta Crystallographica Section E

Structure Reports

Online

ISSN 1600-5368

Katayoun Marjani, ${ }^{a}$ Sian C. Davies, ${ }^{\mathbf{b}}$ Marcus C. Durrant, ${ }^{c}$ David L. Hughes, Nejat Khodamorad ${ }^{\mathrm{a}}$ and Assadolah Samodi ${ }^{a}$

${ }^{\text {a}}$ Faculty of Chemistry, Teacher Training University, 49 Mofateh Avenue, 15614 Tehran, Iran, ${ }^{\mathbf{b}}$ Department of Biological Chemistry, John Innes Centre, Norwich Research Park, Colney, Norwich NR4 7UH, England, and ${ }^{\mathrm{c} C o m p u t a t i o n a l ~ B i o l o g y}$ Group, John Innes Centre, Norwich Research Park, Colney,

Norwich NR4 7UH, England

Correspondence e-mail: sianc.davies@bbsrc.ac.uk

\section{Key indicators}

Single-crystal X-ray study

$T=293 \mathrm{~K}$

Mean $\sigma(\mathrm{C}-\mathrm{C})=0.012 \AA$

$R$ factor $=0.049$

$w R$ factor $=0.092$

Data-to-parameter ratio $=8.5$

For details of how these key indicators were automatically derived from the article, see http://journals.iucr.org/e.

\title{
Bis(2,2'-bipyridine)nitratocopper(II) nitrate
}

The title complex, $\left[\mathrm{Cu}\left(\mathrm{C}_{10} \mathrm{H}_{8} \mathrm{~N}_{2}\right)_{2}\left(\mathrm{NO}_{3}\right)\right] \mathrm{NO}_{3}$, is the first reported unsolvated $\left[\mathrm{Cu}(\text { bipy })_{2}\left(\mathrm{NO}_{3}\right)\right] \mathrm{NO}_{3}$ structure (bipy is $2,2^{\prime}$-bipyridine $)$. The $\mathrm{Cu}^{\mathrm{II}}$ atom of the $\left[\mathrm{Cu}(\text { bipy })_{2}\left(\mathrm{NO}_{3}\right)\right]^{+}$ complex is six-coordinated, forming a distorted octahedral geometry; bond lengths to the $\mathrm{N}$ atoms of the pyridine rings and one of the $\mathrm{O}$ atoms of the chelating $\mathrm{NO}_{3}$ ligand lie in the range 1.975 (5)-2.139 (6) $\AA$, with the second $\mathrm{O}$ atom from the $\mathrm{NO}_{3}$ ligand less tightly coordinated at a distance of 2.520 (6) $\AA$. The geometry of the $\mathrm{CuN}_{2} \mathrm{~N}_{2}{ }_{2} \mathrm{OO}^{\prime}$ chromophore more closely resembles that of $\left[\mathrm{Cu}(\text { bipy })_{2}\left(\mathrm{NO}_{2}\right)\right]^{+}$complexes than previously reported $\left[\mathrm{Cu}(\text { bipy })_{2}\left(\mathrm{NO}_{3}\right)\right]^{+}$structures.

\section{Comment}

The $\mathrm{Cu}$ atom of the title complex, (I), is distorted octahedrally coordinated and is ligated by the four bipyridine $\mathrm{N}$ atoms and a chelating $\mathrm{NO}_{3}$ group, for which one of the O-atom donors lies further from the $\mathrm{Cu}$ atom due to Jahn-Teller distortions (Fig. 1).

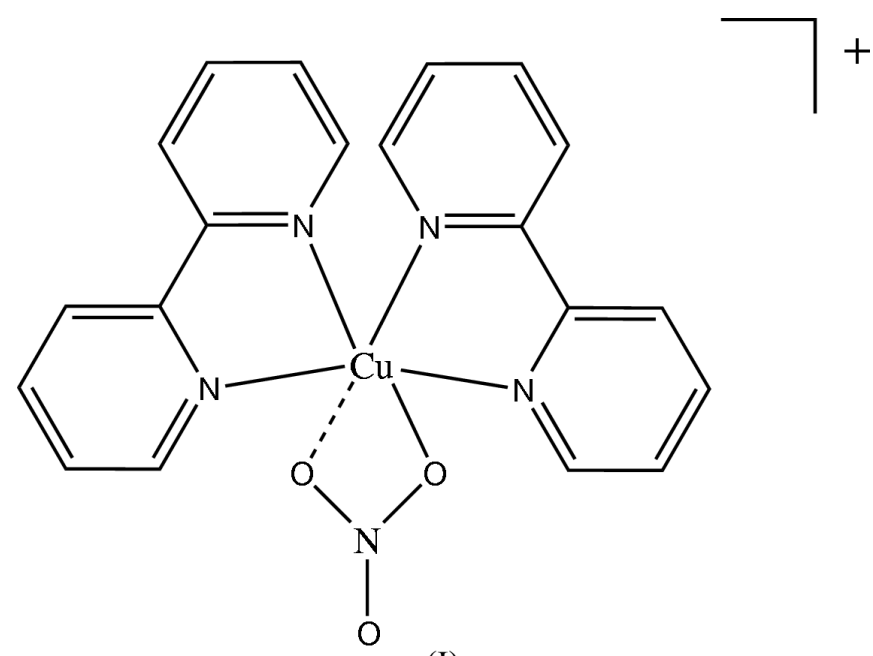

(I)

The $\mathrm{Cu}$ atom has a $\left(4+1^{\prime}+1^{*}\right)$ stereochemistry (Hathaway, 1973 ) with pseudo- $C_{2}$ symmetry bisecting the $\mathrm{NO}_{3}$ ligand and passing between the bipyridine ligands. The atoms of the

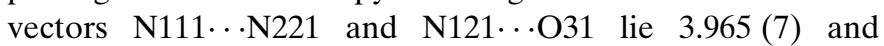
4.009 (8) $\AA$ apart, respectively, and are designated as forming the equatorial plane, with elongation of the N211...O32 distance to 4.512 (8) $\AA$ (designated as the axial atoms). The corresponding $X-\mathrm{Cu}-Y$ angles are also distorted from the ideal octahedral value of $180^{\circ}$, with $\mathrm{N} 111-\mathrm{Cu}-\mathrm{N} 221=$ $176.2(3)^{\circ}, \mathrm{N} 121-\mathrm{Cu}-\mathrm{O} 31=150.0(2)^{\circ}$ and $\mathrm{N} 211-\mathrm{Cu}-\mathrm{O} 32$ $=154.4(2)^{\circ}$. The distortions in the coordination geometry
Received 16 August 2004 Accepted 22 November 2004 Online 4 December 2004 
agree with observations reported (Walsh et al., 1981) for pseudo-Jahn-Teller structures, i.e. as one $\mathrm{Cu}-\mathrm{O}$ bond lengthens, the other shortens, the $\mathrm{Cu}-\mathrm{N}$ bond trans to each $\mathrm{O}$ atom lengthens or shortens, respectively, while the second $\mathrm{Cu}-\mathrm{N}$ bond within the same bipyridine ligand also lengthens or shortens correspondingly but by a smaller amount.

The $\mathrm{Cu}-\mathrm{N}$ bond lengths to the $\mathrm{N}$ atoms in the equatorial plane lie in the range 1.975 (5)-2.013 (6) $\AA$, with the elongated axial $\mathrm{Cu}-\mathrm{N} 211$ bond length being 2.106 (6) $\AA$ (see Table 1); the equatorial $\mathrm{Cu}-\mathrm{O} 31$ bond length is not unusual, being 2.138 (6) $\AA$ (Orpen et al., 1989). The axial $\mathrm{NO}_{3}$ atom $\mathrm{O} 32$ lies 2.520 (6) $\AA$ from the $\mathrm{Cu}$ atom and constitutes the major distortion from regular octahedral coordination. There are no unusual bond dimensions within either the bipyridine ligands or the chelating $\mathrm{NO}_{3}$ ligand, where $\mathrm{N}-\mathrm{O}$ bond lengths lie within the range $1.198(7)-1.257$ (7) $\AA$. Within the nitrate anion, bond lengths lie in the range 1.194 (7)-1.235 (7) $\AA$, as usual for this group.

The coordination geometry about the $\mathrm{Cu}$ atom in (I) is intermediate between reported (Chemical Database Service, Council for the Central Laboratory of the Research Councils, Daresbury Laboratory) $\left[\mathrm{Cu}(\text { bipy })_{2}\left(\mathrm{NO}_{2}\right)\right]^{+}$structures, e.g. $\left[\mathrm{Cu}(\text { bipy) })_{2}\left(\mathrm{NO}_{2}\right)\right] \mathrm{NO}_{3}[(\mathrm{II})$ (Proctor \& Stephens, 1969) and (III) (Simmons et al., 1983, 1987)], $\left[\mathrm{Cu}(\text { bipy })_{2}\left(\mathrm{NO}_{2}\right)\right] \mathrm{BF}_{4}$ [(IV); Walsh et al., 1981], and the four reported [Cu(bipy) $)_{2}(-$ $\left.\left.\mathrm{NO}_{3}\right)\right] \mathrm{NO}_{3}$ structures $\left[\mathrm{Cu}(\text { bipy })_{2}\left(\mathrm{NO}_{3}\right)\right] \mathrm{NO}_{3} \cdot \mathrm{H}_{2} \mathrm{O} \quad[(\mathrm{V})$ (Nakai, 1980), (VI) (Fereday et al., 1981), (VII) (Catalan et al., 1995)] and $\left[\mathrm{Cu}(\text { bipy })_{2}\left(\mathrm{NO}_{3}\right)\right] \mathrm{NO}_{3} \cdot \mathrm{HDCI} \cdot \mathrm{H}_{2} \mathrm{O}[(\mathrm{VIII}) ;$ Prasad et al., 1999; HDCI is 4,5-dicyanoimidazole] (see Table 2). Coordination by the second $\mathrm{O}$ atom in (I) at 2.520 (6) $\AA$ is tighter than in the reported solvated $\left[\mathrm{Cu}(\text { bipy })_{2}\left(\mathrm{NO}_{3}\right)\right]^{+}$ complexes, but is looser than in the $\left[\mathrm{Cu}(\text { bipy })_{2}\left(\mathrm{NO}_{2}\right)\right]^{+}$ complexes at room temperature. However, angles about the $\mathrm{Cu}$ atom in (I) more closely resemble those in the $\mathrm{NO}_{2}$-ligated structures than the $\mathrm{NO}_{3}$-ligated structures, leading to a geometry closer to those in the unsolvated structures.

The crystal packing of the complex in (I) is also similar to that found in the three $\mathrm{NO}_{2}$-ligated structures, with the cations forming corrugated planes seen edge-on when viewed along the crystallographic $c$ axis (Fig. 2). The anions in (I) lie at the apices of the ridges in the cationic 'planes' and form correspondingly corrugated intercationic planes; the anions overlap the ligated $\mathrm{NO}_{3}$ groups to form chains parallel to the crystallographic $c$ axis. This arrangement is as found in the crystal packing of (II), (III) and (IV), where the $\left[\mathrm{BF}_{4}\right]^{-}$anion in (IV) occupies the same relative position as that of the $\left[\mathrm{NO}_{3}\right]^{-}$ anions in (II) and (III). The inclusion of solvent water in the four previously reported $\left[\mathrm{Cu}(\text { bipy })_{2}\left(\mathrm{NO}_{3}\right)\right]^{+}$structures introduces hydrogen bonding between the anion and solvent molecules and the packing arrangements in these crystal structures differ from those of the $\mathrm{NO}_{2}$-ligated complexes. Structures (V), (VI) and (VIII) consist of alternating flat cationic and anionic planes. The ligated $\mathrm{NO}_{3}$ groups in (V) and (VI) lie within the anionic planes, with the water molecules lying within the cationic planes. In (VIII), the HDCI and water molecules all lie within the anionic planes. The packing arrangement in (VII) is different in that the cations form a

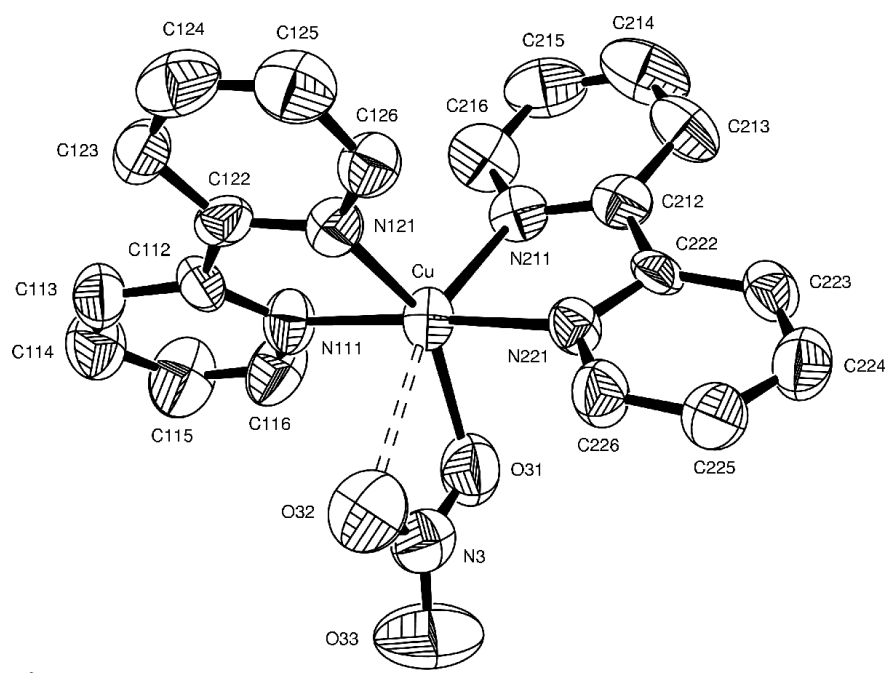

Figure 1

A view of the cation of (I). Displacement ellipsoids are drawn at the $50 \%$ probability level. $\mathrm{H}$ atoms have been omitted for clarity.

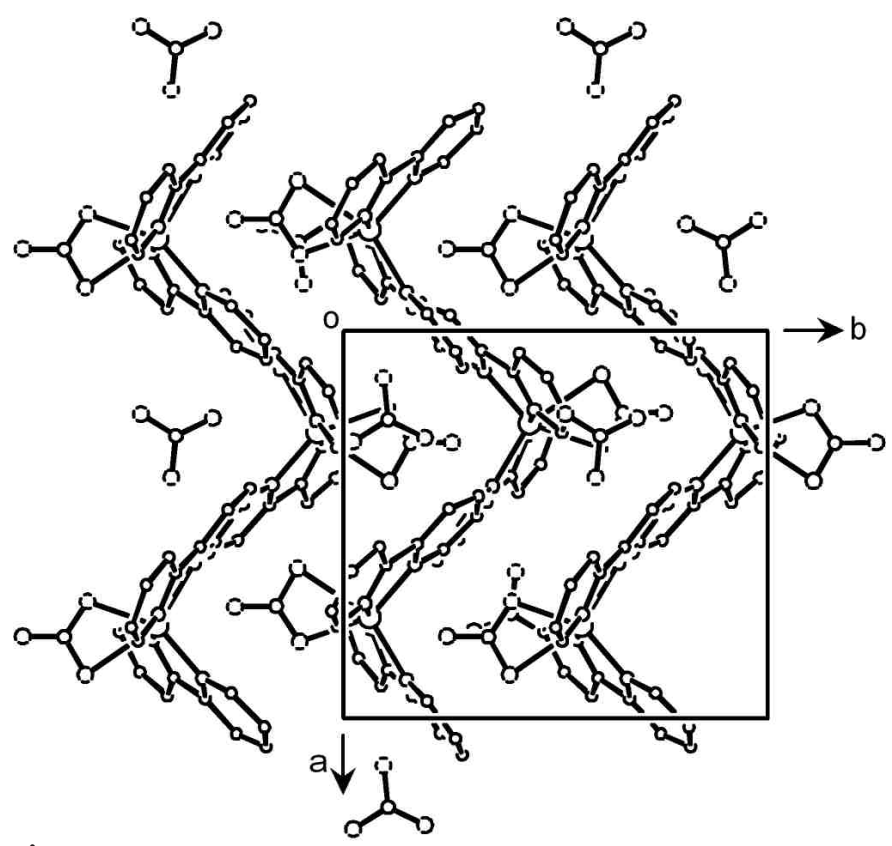

Figure 2

Packing diagram of (I), viewed along the crystallographic $c$ axis. Atoms are represented by arbitrary spheres. $\mathrm{H}$ atoms have been omitted.

three-dimensional framework, with the anions and water molecules lying in planes within this framework.

These results indicate the sensitivity of the $\mathrm{Cu}$ coordination geometry in $\left[\mathrm{Cu}(\text { bipy })_{2}\left(\mathrm{NO}_{3}\right)\right]^{+}$structures to factors such as the identity of the anion and the presence of solvent in the crystal structure. The above examples of $\left[\mathrm{Cu}(\text { bipy })\left(\mathrm{NO}_{2}\right)\right]^{+}$ coordination complexes crystallize in space group No. $14, P 2_{1} /$ $n$, with similar unit cells and crystal packing. Hydrogen bonding in the solvated $\left[\mathrm{Cu}(\text { bipy })_{2}\left(\mathrm{NO}_{3}\right)\right]^{+}$structures, however, leads to different molecular arrangements; most crystallize in space group $P \overline{1}$, with different unit cells but similar packing arrangements.

Complex (I) is the first reported unsolvated $\left[\mathrm{Cu}(\text { bipy })_{2}-\right.$ $\left.\left(\mathrm{NO}_{3}\right)\right] \mathrm{NO}_{3}$ structure and, although the coordination 
geometry may be considered to be similar to that in the structure $\left[\mathrm{Cu}(\text { bipy })_{2}\left(\mathrm{NO}_{3}\right)\right] \mathrm{NO}_{3} \cdot \mathrm{HDCI} \cdot \mathrm{H}_{2} \mathrm{O}$ (Prasad et al., $1999)$, it more closely resembles the $\left[\mathrm{Cu}(\text { bipy })_{2}\left(\mathrm{NO}_{2}\right)\right]^{+}$ structures, both in coordination geometry about the $\mathrm{Cu}$ atom and in having a similar packing arrangement in the same space group, viz. $P 2_{1} / n$.

\section{Experimental}

The preparation of the title compound was carried out under a dinitrogen atmosphere. To a stirred solution of $\left[\mathrm{Cu}\left(\mathrm{NO}_{3}\right)\left(\mathrm{SC}_{5} \mathrm{H}_{4} \mathrm{NH}\right)_{2}\right]$ $(0.26 \mathrm{~g}, 0.74 \mathrm{mmol})$, prepared according to the literature procedure of Davies et al. (1997), in MeOH (25 ml) was added bipyridine (bipy; $0.13 \mathrm{~g}, 1.20 \mathrm{mmol}$ ). The mixture was stirred for $20 \mathrm{~h}$ and then boiled under reflux for $1 \mathrm{~h}$, giving a green solution. This was allowed to cool and was then filtered. The filtrate was concentrated to $c a 4 \mathrm{ml}$ in vacuo, giving a blue solid. This was filtered off, washed with diethyl ether and dried in vacuo as $\left[\mathrm{Cu}(\text { bipy })_{2}\left(\mathrm{NO}_{3}\right)\right] \mathrm{NO}_{3}$ (yield $0.21 \mathrm{~g}$, 80\%). IR: $1600(\mathrm{sh}), 1580(\mathrm{~m}), 1470(\mathrm{~m}), 1320(\mathrm{~m}), 1110(\mathrm{~m}), 830(\mathrm{w})$,

Table 1

Selected geometric parameters $\left(\AA,^{\circ}\right)$.

\begin{tabular}{lrlr}
\hline $\mathrm{Cu}-\mathrm{N} 111$ & $1.975(5)$ & $\mathrm{Cu}-\mathrm{N} 221$ & $1.993(5)$ \\
$\mathrm{Cu}-\mathrm{N} 121$ & $2.013(6)$ & $\mathrm{Cu}-\mathrm{O} 31$ & $2.138(6)$ \\
$\mathrm{Cu}-\mathrm{N} 211$ & $2.106(6)$ & $\mathrm{Cu}-\mathrm{O} 32$ & $2.520(6)$ \\
& & & \\
$\mathrm{N} 111-\mathrm{Cu}-\mathrm{N} 121$ & $80.8(3)$ & $\mathrm{N} 211-\mathrm{Cu}-\mathrm{O} 31$ & $101.2(2)$ \\
$\mathrm{N} 111-\mathrm{Cu}-\mathrm{N} 211$ & $102.8(3)$ & $\mathrm{N} 221-\mathrm{Cu}-\mathrm{O} 31$ & $87.2(2)$ \\
$\mathrm{N} 111-\mathrm{Cu}-\mathrm{N} 221$ & $176.2(3)$ & $\mathrm{N} 111-\mathrm{Cu}-\mathrm{O} 32$ & $83.6(2)$ \\
$\mathrm{N} 121-\mathrm{Cu}-\mathrm{N} 211$ & $108.8(2)$ & $\mathrm{N} 121-\mathrm{Cu}-\mathrm{O} 32$ & $96.7(2)$ \\
$\mathrm{N} 221-\mathrm{Cu}-\mathrm{N} 121$ & $99.9(2)$ & $\mathrm{N} 211-\mathrm{Cu}-\mathrm{O} 32$ & $154.4(2)$ \\
$\mathrm{N} 221-\mathrm{Cu}-\mathrm{N} 211$ & $80.5(2)$ & $\mathrm{N} 221-\mathrm{Cu}-\mathrm{O} 32$ & $92.6(2)$ \\
$\mathrm{N} 111-\mathrm{Cu}-\mathrm{O} 31$ & $90.4(2)$ & $\mathrm{O} 31-\mathrm{Cu}-\mathrm{O} 32$ & $53.57(19)$ \\
$\mathrm{N} 121-\mathrm{Cu}-\mathrm{O} 31$ & $150.0(2)$ & & \\
\hline
\end{tabular}

Crystal data

$\left[\mathrm{Cu}\left(\mathrm{C}_{10} \mathrm{H}_{8} \mathrm{~N}_{2}\right)_{2}\left(\mathrm{NO}_{3}\right)\right] \mathrm{NO}_{3}$

$M_{r}=499.93$

Monoclinic, $P 2_{1} / n$

$a=11.3309(13) \AA$

$b=12.2714(14) \AA$

$c=15.0877(15) \AA$

$\beta=98.281(8)^{\circ}$

$V=2076.0(4) \AA^{3}$

$Z=4$

\section{Data collection}

Enraf-Nonius CAD-4 diffractometer $\omega / \theta$ scans

Absorption correction: $\psi$ scan (EMPABS; Sheldrick et al., 1977)

$T_{\min }=0.751, T_{\max }=0.820$

3287 measured reflections

2546 independent reflections

1128 reflections with $I>2 \sigma(I)$

\section{Refinement}

Refinement on $F^{2}$

$R\left[F^{2}>2 \sigma\left(F^{2}\right)\right]=0.049$

$w R\left(F^{2}\right)=0.092$

$S=0.97$

2546 reflections

298 parameters
$D_{x}=1.600 \mathrm{Mg} \mathrm{m}^{-3}$

Mo $K \alpha$ radiation

Cell parameters from 24

reflections

$\theta=10-11^{\circ}$

$\mu=1.11 \mathrm{~mm}^{-1}$

$T=293(2) \mathrm{K}$

Prism, blue-green Or turquoise? $0.29 \times 0.21 \times 0.18 \mathrm{~mm}$ $770(s), 730(m), 630(w), 415(w), 290(w) \mathrm{cm}^{-1}$. Recrystallization of (I) by slow diffusion of diethyl ether into a methanol solution gave turquoise-coloured crystals.

Table 2

Comparison of bond dimensions $\left(\AA{ }^{\circ},\right)$ for $(\mathrm{I})$ and related structures.

\begin{tabular}{|c|c|c|c|c|c|c|c|c|}
\hline & (II) & (III) & (IV) & (I) & $(\mathrm{V})$ & (VI) & $\begin{array}{l}\text { (VII) } \\
\text { Molecule 1/2 }\end{array}$ & (VIII) \\
\hline $\mathrm{Cu}-\mathrm{N}$ & $1.980(11)$ & $1.980(3)$ & $1.990(5)$ & $1.975(5)$ & $1.984(5)$ & $1.986(5)$ & $1.974(3) / 1.969(3)$ & $1.980(4)$ \\
\hline $\mathrm{Cu}-\mathrm{N}_{\mathrm{O}}$ & $2.065(10)$ & $2.074(4)$ & $2.052(5)$ & $2.013(6)$ & $2.022(5)$ & $2.023(5)$ & $2.021(3) / 2.036(3)$ & $2.032(3)$ \\
\hline $\mathrm{Cu}-\mathrm{N}^{*}$ & $2.006(10)$ & $1.988(3)$ & $2.004(5)$ & $1.993(5)$ & $1.982(5)$ & $1.973(5)$ & $1.978(3) / 1.981(3)$ & $2.008(4)$ \\
\hline $\mathrm{Cu}-\mathrm{N}_{\mathrm{O}} *$ & $2.100(9)$ & $2.085(4)$ & $2.142(5)$ & $2.106(6)$ & $2.045(5)$ & $2.051(5)$ & $2.109(3) / 2.097(3)$ & $2.185(3)$ \\
\hline $\mathrm{Cu}-\mathrm{O}$ & $2.238(10)$ & $2.230(5)$ & $2.117(6)$ & $2.138(6)$ & $2.299(7)$ & $2.301(5)$ & $2.116^{a} / 2.184(3)$ & $2.078(3)$ \\
\hline $\mathrm{Cu}-\mathrm{O}^{*}$ & $2.329(10)$ & $2.320(5)$ & $2.462(6)$ & $2.520(6)$ & $2.818(7)$ & $2.832(5)$ & $2.822(4) / 2.717(3)$ & $2.639(4)$ \\
\hline $\mathrm{O}-\mathrm{Cu}-\mathrm{N}_{\mathrm{O}}$ & $157.8(4)$ & $157.7(2)$ & $164.1(1)$ & $150.0(2)$ & $127.8(3)$ & $127.5(2)$ & $143.77(13) / 135.28(12)$ & $161.5(1)$ \\
\hline $\mathrm{O}^{*}-\mathrm{Cu}-\mathrm{N}_{\mathrm{O}}^{*}$ & $151.1(4)$ & $151.3(2)$ & $149.2(1)$ & $154.4(2)$ & $139.5(4)$ & $139.2(1)$ & $138.11(12) / 144.49(11)$ & $141.8^{\mathrm{a}, b}$ \\
\hline $\mathrm{N}-\mathrm{Cu}-\mathrm{N}^{*}$ & $179.6(4)$ & $179.7(2)$ & $178.6(1)$ & $176.2(3)$ & $170.9(3)$ & $170.7(1)$ & $177.05(14) / 177.46(13)$ & $176.7(1)$ \\
\hline $\mathrm{O}-\mathrm{Cu}-\mathrm{O}^{*}$ & $52.5(4)$ & $52.8(2)$ & $52.7(2)$ & $53.6(2)$ & $47.7(4)$ & $47.7(1)$ & $48.25(11) / 50.66(10)$ & $53.0^{a, b}$ \\
\hline $\mathrm{O}-\mathrm{Cu}-\mathrm{N}_{\mathrm{O}} *$ & $99.2(4)$ & $99.3(2)$ & $97.3(2)$ & $101.2(2)$ & $92.1(3)$ & $91.8(2)$ & $90.64(12) / 94.69(11)$ & $90.8(1)$ \\
\hline $\mathrm{O}^{*}-\mathrm{Cu}-\mathrm{N}_{\mathrm{O}}$ & $105.6(4)$ & $105.4(1)$ & $111.9(2)$ & $96.7(2)$ & $80.3(4)$ & $80.0(1)$ & $96.10(12) / 85.13(11)$ & $109.5^{a, b}$ \\
\hline $\mathrm{N}_{\mathrm{O}}-\mathrm{Cu}-\mathrm{N}_{\mathrm{O}} *$ & $103.0(4)$ & $102.8(1)$ & $98.5(2)$ & $108.8(2)$ & $140.2(3)$ & $140.7(1)$ & $125.52(13) / 130.01(13)$ & $107.5(1)$ \\
\hline $\mathrm{O}-\mathrm{Cu}-\mathrm{N}$ & $93.5(4)$ & $93.7(2)$ & $94.1(2)$ & $90.4(2)$ & $86.7(3)$ & $86.3(2)$ & $88.86(13) / 89.74(12)$ & $92.5(1)$ \\
\hline $\mathrm{O}-\mathrm{Cu}-\mathrm{N}^{*}$ & $86.8(4)$ & $86.5(2)$ & $87.2(2)$ & $87.2(2)$ & $85.5(3)$ & $85.5(2)$ & $89.88(13) / 88.00(12)$ & $88.1(1)$ \\
\hline $\mathrm{O}^{*}-\mathrm{Cu}-\mathrm{N}$ & $89.1(4)$ & $89.3(1)$ & $89.5(2)$ & $83.6(2)$ & $81.8(4)$ & $81.6(1)^{b}$ & $86.56(13) / 88.14$ (12) & $90.6^{a, b}$ \\
\hline $\mathrm{O}^{*}-\mathrm{Cu}-\mathrm{N}^{*}$ & $91.2(4)$ & $90.7(2)$ & $90.9(2)$ & $92.6(2)$ & $89.5(4)$ & $89.6(1)^{b}$ & $90.62(14) / 89.53(12)$ & $87.3^{a, b}$ \\
\hline $\mathrm{N}-\mathrm{Cu}-\mathrm{N}_{\mathrm{O}}$ & $81.1(4)$ & $80.0(2)$ & $80.8(2)$ & $80.8(3)$ & $81.1(3)$ & $81.5(2)$ & $81.60(14) / 81.40(13)$ & $81.1(1)$ \\
\hline $\mathrm{N}-\mathrm{Cu}-\mathrm{N}_{\mathrm{O}} *$ & $99.4(4)$ & $100.6(1)$ & $101.0(2)$ & $102.8(3)$ & $103.6(3)$ & $103.7(2)$ & $102.55(13) / 100.72(13)$ & $104.2(1)$ \\
\hline $\mathrm{N}^{*}-\mathrm{Cu}-\mathrm{N}_{\mathrm{O}}$ & $98.7(4)$ & $99.8(2)$ & $97.8(2)$ & $99.9(2)$ & $100.0(3)$ & $100.0(2)$ & $97.88(14) / 99.40(13)$ & $97.4(1)$ \\
\hline $\mathrm{N}^{*}-\mathrm{Cu}-\mathrm{N}_{\mathrm{O}} *$ & $80.3(4)$ & $79.6(2)$ & $79.4(2)$ & $80.5(2)$ & $81.4(3)$ & $81.0(2)$ & $80.13(13) / 80.63$ (13) & $79.0(1)$ \\
\hline
\end{tabular}

Notes: (a) s.u. values not reported; (b) value was not reported and was calculated using GEOM (Owen, 1981); $\mathrm{N}_{\mathrm{O}}$ denotes $\mathrm{N}$ trans to an $\mathrm{O}$ atom; * denotes the loosely coordinated axial $\mathrm{O}$ atom, the axial $\mathrm{N}$ atom trans to it and the second (equatorial) $\mathrm{N}$ atom within the same bipyridine ligand.

$\mathrm{H}$ atoms were geometrically constrained to ride on the parent atoms $(\mathrm{C}-\mathrm{H}$ $=0.93 \AA$ ), with $U_{\text {iso }}(\mathrm{H})=$ $1.2 U_{\text {eq }}$ (parent atom).

Data collection: $C A D-4$ EXPRESS (Enraf-Nonius, 1992); cell refinement: CAD-4 EXPRESS; data reduction: $C A D-4$ processing program (Hursthouse, 1976); $\operatorname{program}(\mathrm{s})$ used to solve structure: SHELXS86 (Sheldrick, 1985); program(s) used to refine structure: SHELXL97 (Sheldrick, 1997); molecular graphics: ORTEP (Johnson, 1971) and ORTEP-3 (Farrugia, 1997); software used to prepare material for publication: SHELXL97. 


\section{metal-organic papers}

KM thanks FOCTTU, Tehran, for financial support.

\section{References}

Catalan, K. J., Jackson, S., Zubkowski, J. D., Perry, D. L., Valente, E. J., Feliu, L. A. \& Polanco, A. (1995). Polyhedron, 14, 2165-2171.

Davies, S. C., Durrant, M. C., Hughes, D. L., Leidenberger, K., Stapper, C. \& Richards, R. L. (1997). J. Chem. Soc. Dalton Trans. pp. 2409-2418.

Enraf-Nonius (1992). CAD-4 EXPRESS. Enraf-Nonius, Delft, The Netherlands.

Farrugia, L. J. (1997). J. Appl. Cryst. 30, 565.

Fereday, R. J., Hodgson, P., Tyagi, S. \& Hathaway, B. J. (1981). J. Chem. Soc. Dalton Trans. pp. 2070-2077.

Hathaway, B. J. (1973). Struct. Bonding (Berlin), 14, 49-67.

Hursthouse, M. B. (1976). CAD-4 processing program. Queen Mary College, London.

Johnson, C. K. (1971). ORTEPII. Report ORNL-3794, revised. Oak Ridge National Laboratory, Tennessee, USA.
Nakai, H. (1980). Bull. Chem. Soc. Jpn, 53, 1321-1326.

Orpen, A. G., Brammer, L., Allen, F. H., Kennard, O., Watson, D. G. \& Taylor, R. (1989). J. Chem. Soc. Dalton Trans. pp. S1-83.

Owen, J. D. (1981). GEOM. Rothamsted Experimental Station, Harpenden, Herts, England.

Prasad, B. L. V., Sato, H., Enoki, T., Cohen, S. \& Radhakrishnan, T. P. (1999). J. Chem. Soc. Dalton Trans. pp. 25-29.

Proctor, I. M. \& Stephens, F. S. (1969). J. Chem. Soc. A, pp. 1248-1256.

Sheldrick, G. M., Orpen, A. G., Reichert, B. E. \& Raithby, P. R. (1977). $E M P A B S$. 4th European Crystallographic Meeting, Oxford, Abstracts, p. 147.

Sheldrick, G. M. (1985). SHELXS86. University of Göttingen, Germany.

Sheldrick, G. M. (1997). SHELXL97. University of Göttingen, Germany.

Simmons, C. J., Clearfield, A., Fitzgerald, W., Tyagi, S. \& Hathaway, B. (1983). J. Chem. Soc. Chem. Commun. pp. 189-190.

Simmons, C. J., Hathaway, B. J., Amornjarusiri, K., Santarsiero, B. D. \& Clearfield, A. (1987). J. Am. Chem. Soc. 109, 1947-1958.

Walsh, A., Walsh, B., Murphy, B. \& Hathaway, B. J. (1981). Acta Cryst. B37, $1512-1520$. 


\section{supporting information}

Acta Cryst. (2005). E61, m11-m14 [https://doi.org/10.1107/S1600536804030788]

\section{Bis(2,2'-bipyridine)nitratocopper(II) nitrate}

\section{Katayoun Marjani, Sian C. Davies, Marcus C. Durrant, David L. Hughes, Nejat Khodamorad and Assadolah Samodi}

Bis(2,2'-bipyridine)nitratocopper(II) nitrate

Crystal data

$\left[\mathrm{Cu}\left(\mathrm{C}_{10} \mathrm{H}_{8} \mathrm{~N}_{2}\right)_{2}\left(\mathrm{NO}_{3}\right)\right] \mathrm{NO}_{3}$

$F(000)=1020$

$M_{r}=499.93$

Monoclinic, $P 2_{1} / n$

Hall symbol: -P 2 yn

$a=11.3309$ (13) $\AA$

$b=12.2714(14) \AA$

$c=15.0877(15) \AA$

$\beta=98.281(8)^{\circ}$

$V=2076.0(4) \AA^{3}$

$Z=4$

$D_{\mathrm{x}}=1.600 \mathrm{Mg} \mathrm{m}^{-3}$

Mo $K \alpha$ radiation, $\lambda=0.71069 \AA$

Cell parameters from 24 reflections

$\theta=10-11^{\circ}$

$\mu=1.11 \mathrm{~mm}^{-1}$

$T=293 \mathrm{~K}$

Prism, blue green

$0.29 \times 0.21 \times 0.18 \mathrm{~mm}$

\section{Data collection}

Enraf-Nonius CAD-4 diffractometer

Radiation source: fine-focus sealed tube

2546 independent reflections

Graphite monochromator

scintillation counter; $\omega / \theta$ scans

Absorption correction: $\psi$ scan

(EMPABS; Sheldrick et al., 1977)

$T_{\min }=0.751, T_{\max }=0.820$

3287 measured reflections

1128 reflections with $I>2 \sigma(I)$

$R_{\text {int }}=0.028$

$\theta_{\max }=23.0^{\circ}, \theta_{\min }=1.5^{\circ}$

$h=-11 \rightarrow 11$

$k=-1 \rightarrow 12$

$l=-1 \rightarrow 15$

3 standard reflections every $167 \mathrm{~min}$

intensity decay: $2.0 \%$

\section{Refinement}

Refinement on $F^{2}$

Least-squares matrix: full

$R\left[F^{2}>2 \sigma\left(F^{2}\right)\right]=0.049$

$w R\left(F^{2}\right)=0.092$

$S=0.97$

2546 reflections

298 parameters

0 restraints

Primary atom site location: structure-invariant direct methods

Secondary atom site location: difference Fourier map

Hydrogen site location: inferred from neighbouring sites

$\mathrm{H}$-atom parameters constrained

$w=\sigma^{-2}\left(F_{\mathrm{o}}^{2}\right)$

$(\Delta / \sigma)_{\max }=0.001$

$\Delta \rho_{\max }=0.27 \mathrm{e}^{-3}$

$\Delta \rho_{\min }=-0.28$ e $\AA^{-3}$ 


\section{Special details}

Geometry. All e.s.d.'s (except the e.s.d. in the dihedral angle between two 1.s. planes) are estimated using the full covariance matrix. The cell e.s.d.'s are taken into account individually in the estimation of e.s.d.'s in distances, angles and torsion angles; correlations between e.s.d.'s in cell parameters are only used when they are defined by crystal symmetry. An approximate (isotropic) treatment of cell e.s.d.'s is used for estimating e.s.d.'s involving 1.s. planes.

Refinement. Refinement of $F^{2}$ against ALL reflections. The weighted $R$-factor $w R$ and goodness of fit $S$ are based on $F^{2}$, conventional $R$-factors $R$ are based on $F$, with $F$ set to zero for negative $F^{2}$. The threshold expression of $F^{2}>\sigma\left(F^{2}\right)$ is used only for calculating $R$-factors (gt) etc. and is not relevant to the choice of reflections for refinement. $R$-factors based on $F^{2}$ are statistically about twice as large as those based on $F$, and $R$ - factors based on ALL data will be even larger.

3287 reflections were collected to $\theta_{\max }$ of $23^{\circ}\left(\mathrm{h}_{\max }, \mathrm{k}_{\max }, 1_{\max }\right.$ of $\left.12,13,16\right)$, with 2887 unique reflections and 1172 observed. Those greater than $22^{\circ}$, however, were found to be too unreliable and were not used in the final refinement, leaving 2546 unique reflections and 1128 observed. $\mathrm{H}$ atoms were geometrically constrained to ride on the parent atoms, with isotropic displacement parameters set to be $1.2 U_{\mathrm{eq}}$ of the parent atom. Data were corrected for Lorentz-polarization effects, decay of the intensities (Hursthouse, 1976), absorption (Sheldrick et al., 1977) and negative intensities (French et al., 1978) before structure solution and refinement.

French, S. \& Wilson, K. (1978). Acta Cryst. A34, 517-525.

Fractional atomic coordinates and isotropic or equivalent isotropic displacement parameters $\left(\AA^{2}\right)$

\begin{tabular}{|c|c|c|c|c|}
\hline & $x$ & $y$ & $z$ & $U_{\text {iso }} * / U_{\text {eq }}$ \\
\hline $\mathrm{Cu}$ & $0.23885(8)$ & $0.43850(8)$ & $0.12480(6)$ & $0.0508(3)$ \\
\hline N111 & $0.2036(5)$ & $0.4497(5)$ & $0.2489(4)$ & $0.0543(16)$ \\
\hline C112 & $0.1041(6)$ & 0.3999 (6) & $0.2653(5)$ & $0.046(2)$ \\
\hline $\mathrm{C} 113$ & $0.0581(6)$ & $0.4147(7)$ & $0.3445(5)$ & $0.063(2)$ \\
\hline H113 & -0.0127 & 0.3814 & 0.3541 & $0.076^{*}$ \\
\hline $\mathrm{C} 114$ & $0.1197(8)$ & $0.4796(7)$ & $0.4076(6)$ & $0.074(3)$ \\
\hline H114 & 0.0904 & 0.4914 & 0.4613 & $0.089 *$ \\
\hline C115 & $0.2234(8)$ & $0.5277(7)$ & $0.3939(6)$ & $0.087(3)$ \\
\hline H115 & 0.2676 & 0.5685 & 0.4390 & $0.104 *$ \\
\hline $\mathrm{C} 116$ & $0.2619(7)$ & $0.5149(7)$ & $0.3120(6)$ & $0.076(3)$ \\
\hline H116 & 0.3295 & 0.5521 & 0.3002 & $0.091 *$ \\
\hline N121 & $0.1031(5)$ & $0.3316(5)$ & $0.1175(4)$ & $0.0471(17)$ \\
\hline $\mathrm{C} 122$ & $0.0503(6)$ & $0.3258(6)$ & $0.1924(5)$ & 0.048 (2) \\
\hline C123 & $-0.0407(6)$ & $0.2512(6)$ & $0.2003(6)$ & $0.057(2)$ \\
\hline H123 & -0.0776 & 0.2486 & 0.2514 & $0.069^{*}$ \\
\hline $\mathrm{C} 124$ & $-0.0733(7)$ & $0.1822(7)$ & $0.1301(7)$ & $0.073(3)$ \\
\hline H124 & -0.1322 & 0.1306 & 0.1347 & $0.087 *$ \\
\hline $\mathrm{C} 125$ & $-0.0226(7)$ & $0.1861(7)$ & $0.0535(6)$ & $0.070(3)$ \\
\hline H125 & -0.0454 & 0.1390 & 0.0059 & $0.084 *$ \\
\hline C126 & $0.0649(6)$ & $0.2647(6)$ & $0.0509(5)$ & $0.053(2)$ \\
\hline H126 & 0.0990 & 0.2707 & -0.0014 & $0.064 *$ \\
\hline N211 & $0.4007(5)$ & $0.3514(5)$ & $0.1403(4)$ & $0.0487(16)$ \\
\hline C212 & $0.4419(6)$ & $0.3379(6)$ & $0.0608(6)$ & $0.051(2)$ \\
\hline C213 & $0.5386(7)$ & $0.2717(7)$ & $0.0557(6)$ & $0.071(3)$ \\
\hline $\mathrm{H} 213$ & 0.5665 & 0.2623 & 0.0012 & $0.085^{*}$ \\
\hline $\mathrm{C} 214$ & $0.5936(8)$ & $0.2200(7)$ & $0.1300(8)$ & $0.092(4)$ \\
\hline $\mathrm{H} 214$ & 0.6582 & 0.1743 & 0.1264 & $0.111 *$ \\
\hline $\mathrm{C} 215$ & $0.5530(9)$ & $0.2359(7)$ & $0.2105(7)$ & $0.085(3)$ \\
\hline $\mathrm{H} 215$ & 0.5904 & 0.2019 & 0.2621 & $0.101 *$ \\
\hline
\end{tabular}




$\begin{array}{lllll}\mathrm{C} 216 & 0.4556(7) & 0.3029(7) & 0.2139(6) & 0.072(3) \\ \mathrm{H} 216 & 0.4281 & 0.3143 & 0.2683 & 0.087^{*} \\ \mathrm{~N} 221 & 0.2685(5) & 0.4364(5) & -0.0022(3) & 0.0437(14) \\ \mathrm{C} 222 & 0.3758(6) & 0.3955(5) & -0.0144(5) & 0.0402(18) \\ \mathrm{C} 223 & 0.4158(6) & 0.4111(6) & -0.0968(5) & 0.052(2) \\ \mathrm{H} 223 & 0.4903 & 0.3853 & -0.1058 & 0.062^{*} \\ \mathrm{C} 224 & 0.3446(8) & 0.4647(7) & -0.1649(5) & 0.067(2) \\ \mathrm{H} 224 & 0.3713 & 0.4768 & -0.2195 & 0.080^{*} \\ \mathrm{C} 225 & 0.2346(8) & 0.4997(6) & -0.1510(5) & 0.067(2) \\ \mathrm{H} 225 & 0.1842 & 0.5340 & -0.1968 & 0.081^{*} \\ \mathrm{C} 226 & 0.1994(6) & 0.4840(6) & -0.0696(5) & 0.058(2) \\ \mathrm{H} 226 & 0.1240 & 0.5076 & -0.0608 & 0.069^{*} \\ \mathrm{~N} 3 & 0.2103(7) & 0.6582(7) & 0.1169(4) & 0.063(2) \\ \mathrm{O} 31 & 0.3019(5) & 0.6029(5) & 0.1307(4) & 0.0780(19) \\ \mathrm{O} 32 & 0.1127(5) & 0.6081(5) & 0.1012(4) & 0.090(2) \\ \mathrm{O} 33 & 0.2120(6) & 0.7558(5) & 0.1158(5) & 0.124(3) \\ \mathrm{N} 4 & 0.7290(7) & 0.3951(7) & 0.3817(5) & 0.068(2) \\ \mathrm{O} 41 & 0.7854(5) & 0.4768(5) & 0.3685(4) & 0.0811(19) \\ \mathrm{O} 42 & 0.6221(5) & 0.4057(5) & 0.3877(4) & 0.091(2) \\ \mathrm{O} 43 & 0.7756(6) & 0.3078(5) & 0.3912(5) & 0.126(3)\end{array}$

Atomic displacement parameters $\left(\AA^{2}\right)$

\begin{tabular}{lllllll}
\hline & $U^{11}$ & $U^{22}$ & $U^{33}$ & $U^{12}$ & $U^{13}$ & $U^{23}$ \\
\hline $\mathrm{Cu}$ & $0.0470(5)$ & $0.0616(6)$ & $0.0451(5)$ & $-0.0043(6)$ & $0.0114(4)$ & $-0.0019(6)$ \\
$\mathrm{N} 111$ & $0.056(4)$ & $0.070(5)$ & $0.037(4)$ & $-0.006(4)$ & $0.011(3)$ & $-0.006(4)$ \\
$\mathrm{C} 112$ & $0.043(5)$ & $0.049(5)$ & $0.050(5)$ & $0.008(4)$ & $0.015(4)$ & $-0.007(4)$ \\
$\mathrm{C} 113$ & $0.054(5)$ & $0.073(7)$ & $0.066(6)$ & $0.008(5)$ & $0.021(5)$ & $0.009(5)$ \\
$\mathrm{C} 114$ & $0.082(7)$ & $0.084(7)$ & $0.057(6)$ & $0.001(6)$ & $0.017(5)$ & $-0.004(5)$ \\
$\mathrm{C} 115$ & $0.102(8)$ & $0.103(8)$ & $0.057(7)$ & $-0.034(6)$ & $0.013(5)$ & $-0.024(5)$ \\
$\mathrm{C} 116$ & $0.085(6)$ & $0.084(7)$ & $0.060(7)$ & $-0.028(5)$ & $0.011(5)$ & $-0.001(6)$ \\
$\mathrm{N} 121$ & $0.042(4)$ & $0.051(4)$ & $0.048(4)$ & $-0.002(3)$ & $0.005(3)$ & $0.003(4)$ \\
$\mathrm{C} 122$ & $0.040(5)$ & $0.042(5)$ & $0.061(6)$ & $0.006(4)$ & $0.006(4)$ & $0.016(5)$ \\
$\mathrm{C} 123$ & $0.048(5)$ & $0.061(6)$ & $0.065(6)$ & $0.000(5)$ & $0.015(5)$ & $0.018(5)$ \\
$\mathrm{C} 124$ & $0.064(6)$ & $0.056(6)$ & $0.093(8)$ & $-0.018(5)$ & $-0.005(6)$ & $0.001(6)$ \\
$\mathrm{C} 125$ & $0.066(6)$ & $0.066(7)$ & $0.075(7)$ & $-0.010(5)$ & $-0.001(5)$ & $-0.010(6)$ \\
$\mathrm{C} 126$ & $0.048(5)$ & $0.056(6)$ & $0.056(6)$ & $-0.008(5)$ & $0.006(4)$ & $-0.001(5)$ \\
$\mathrm{N} 211$ & $0.040(4)$ & $0.051(4)$ & $0.052(4)$ & $-0.004(3)$ & $-0.004(4)$ & $0.000(4)$ \\
$\mathrm{C} 212$ & $0.038(5)$ & $0.046(5)$ & $0.067(6)$ & $-0.009(4)$ & $0.003(4)$ & $-0.008(5)$ \\
$\mathrm{C} 213$ & $0.051(6)$ & $0.073(7)$ & $0.087(7)$ & $0.020(5)$ & $0.000(5)$ & $-0.017(6)$ \\
$\mathrm{C} 214$ & $0.054(6)$ & $0.085(8)$ & $0.129(10)$ & $0.027(5)$ & $-0.020(7)$ & $-0.024(8)$ \\
$\mathrm{C} 215$ & $0.081(8)$ & $0.067(7)$ & $0.097(9)$ & $0.007(6)$ & $-0.019(6)$ & $0.017(6)$ \\
$\mathrm{C} 216$ & $0.067(6)$ & $0.081(7)$ & $0.063(7)$ & $0.005(5)$ & $-0.013(5)$ & $-0.005(5)$ \\
$\mathrm{N} 221$ & $0.049(4)$ & $0.050(4)$ & $0.031(4)$ & $0.006(4)$ & $0.005(3)$ & $-0.005(3)$ \\
$\mathrm{C} 222$ & $0.052(5)$ & $0.029(4)$ & $0.041(5)$ & $0.006(4)$ & $0.009(4)$ & $-0.011(4)$ \\
$\mathrm{C} 223$ & $0.053(5)$ & $0.051(6)$ & $0.052(5)$ & $-0.006(4)$ & $0.014(4)$ & $-0.016(5)$ \\
$\mathrm{C} 224$ & $0.085(6)$ & $0.067(7)$ & $0.053(6)$ & $-0.017(5)$ & $0.025(5)$ & $-0.008(5)$ \\
$\mathrm{C} 225$ & $0.089(7)$ & $0.064(6)$ & $0.051(6)$ & $0.011(5)$ & $0.016(5)$ & $0.005(4)$ \\
& & & & & &
\end{tabular}




\begin{tabular}{lllllll}
$\mathrm{C} 226$ & $0.065(5)$ & $0.068(6)$ & $0.042(5)$ & $0.011(4)$ & $0.011(5)$ & $0.009(5)$ \\
$\mathrm{N} 3$ & $0.090(7)$ & $0.061(6)$ & $0.040(4)$ & $-0.004(5)$ & $0.008(5)$ & $-0.003(5)$ \\
$\mathrm{O} 31$ & $0.067(4)$ & $0.094(5)$ & $0.077(4)$ & $0.014(4)$ & $0.024(3)$ & $0.015(4)$ \\
$\mathrm{O} 32$ & $0.082(4)$ & $0.097(5)$ & $0.089(5)$ & $-0.014(4)$ & $0.005(4)$ & $0.000(4)$ \\
$\mathrm{O} 33$ & $0.166(7)$ & $0.054(4)$ & $0.143(6)$ & $-0.007(5)$ & $-0.013(5)$ & $-0.012(5)$ \\
$\mathrm{N} 4$ & $0.087(7)$ & $0.063(6)$ & $0.055(5)$ & $-0.006(6)$ & $0.012(5)$ & $0.007(5)$ \\
$\mathrm{O} 41$ & $0.081(5)$ & $0.068(4)$ & $0.097(5)$ & $0.001(3)$ & $0.021(4)$ & $0.012(4)$ \\
O42 & $0.078(4)$ & $0.096(5)$ & $0.103(5)$ & $-0.013(4)$ & $0.031(4)$ & $0.000(4)$ \\
O43 & $0.132(6)$ & $0.048(4)$ & $0.198(8)$ & $0.027(4)$ & $0.026(5)$ & $0.032(5)$ \\
\hline
\end{tabular}

Geometric parameters $\left(\AA,{ }^{\circ}\right)$

\begin{tabular}{|c|c|c|c|}
\hline $\mathrm{Cu}-\mathrm{N} 111$ & $1.975(5)$ & $\mathrm{N} 211-\mathrm{C} 216$ & $1.333(8)$ \\
\hline $\mathrm{Cu}-\mathrm{N} 121$ & $2.013(6)$ & $\mathrm{N} 211-\mathrm{C} 212$ & $1.359(8)$ \\
\hline $\mathrm{Cu}-\mathrm{N} 211$ & $2.106(6)$ & $\mathrm{C} 212-\mathrm{C} 213$ & $1.374(9)$ \\
\hline $\mathrm{Cu}-\mathrm{N} 221$ & $1.993(5)$ & $\mathrm{C} 212-\mathrm{C} 222$ & $1.450(9)$ \\
\hline $\mathrm{Cu}-\mathrm{O} 31$ & $2.138(6)$ & $\mathrm{C} 213-\mathrm{C} 214$ & $1.359(11)$ \\
\hline $\mathrm{Cu}-\mathrm{O} 32$ & $2.520(6)$ & $\mathrm{C} 213-\mathrm{H} 213$ & 0.930 \\
\hline $\mathrm{N} 111-\mathrm{C} 112$ & $1.337(7)$ & $\mathrm{C} 214-\mathrm{C} 215$ & $1.373(11)$ \\
\hline $\mathrm{N} 111-\mathrm{C} 116$ & $1.342(8)$ & С214-H214 & 0.930 \\
\hline $\mathrm{C} 112-\mathrm{C} 113$ & $1.383(9)$ & $\mathrm{C} 215-\mathrm{C} 216$ & $1.382(10)$ \\
\hline $\mathrm{C} 112-\mathrm{C} 122$ & $1.488(9)$ & $\mathrm{C} 215-\mathrm{H} 215$ & 0.930 \\
\hline C113-C114 & $1.355(9)$ & $\mathrm{C} 216-\mathrm{H} 216$ & 0.930 \\
\hline C113-H113 & 0.930 & $\mathrm{~N} 221-\mathrm{C} 226$ & $1.326(7)$ \\
\hline $\mathrm{C} 114-\mathrm{C} 115$ & $1.357(9)$ & $\mathrm{N} 221-\mathrm{C} 222$ & $1.352(7)$ \\
\hline C114-H114 & 0.930 & $\mathrm{C} 222-\mathrm{C} 223$ & $1.397(9)$ \\
\hline $\mathrm{C} 115-\mathrm{C} 116$ & $1.378(10)$ & $\mathrm{C} 223-\mathrm{C} 224$ & $1.378(9)$ \\
\hline C115-H115 & 0.930 & $\mathrm{C} 223-\mathrm{H} 223$ & 0.930 \\
\hline $\mathrm{C} 116-\mathrm{H} 116$ & 0.930 & $\mathrm{C} 224-\mathrm{C} 225$ & $1.362(9)$ \\
\hline $\mathrm{N} 121-\mathrm{C} 126$ & $1.322(8)$ & $\mathrm{C} 224-\mathrm{H} 224$ & 0.930 \\
\hline $\mathrm{N} 121-\mathrm{C} 122$ & $1.354(8)$ & $\mathrm{C} 225-\mathrm{C} 226$ & $1.359(9)$ \\
\hline $\mathrm{C} 122-\mathrm{C} 123$ & $1.396(9)$ & $\mathrm{C} 225-\mathrm{H} 225$ & 0.930 \\
\hline $\mathrm{C} 123-\mathrm{C} 124$ & $1.365(9)$ & $\mathrm{C} 226-\mathrm{H} 226$ & 0.930 \\
\hline $\mathrm{C} 123-\mathrm{H} 123$ & 0.930 & $\mathrm{~N} 3-\mathrm{O} 33$ & $1.198(7)$ \\
\hline $\mathrm{C} 124-\mathrm{C} 125$ & $1.363(10)$ & $\mathrm{N} 3-\mathrm{O} 31$ & $1.233(7)$ \\
\hline $\mathrm{C} 124-\mathrm{H} 124$ & 0.930 & $\mathrm{~N} 3-\mathrm{O} 32$ & $1.257(7)$ \\
\hline $\mathrm{C} 125-\mathrm{C} 126$ & $1.388(9)$ & $\mathrm{N} 4-\mathrm{O} 43$ & $1.194(7)$ \\
\hline C125-H125 & 0.930 & $\mathrm{~N} 4-\mathrm{O} 41$ & $1.220(7)$ \\
\hline $\mathrm{C} 126-\mathrm{H} 126$ & 0.930 & $\mathrm{~N} 4-\mathrm{O} 42$ & $1.235(7)$ \\
\hline $\mathrm{N} 111-\mathrm{Cu}-\mathrm{N} 121$ & $80.8(3)$ & $\mathrm{N} 121-\mathrm{C} 126-\mathrm{C} 125$ & $124.5(8)$ \\
\hline $\mathrm{N} 111-\mathrm{Cu}-\mathrm{N} 211$ & $102.8(3)$ & $\mathrm{N} 121-\mathrm{C} 126-\mathrm{H} 126$ & 117.8 \\
\hline $\mathrm{N} 111-\mathrm{Cu}-\mathrm{N} 221$ & $176.2(3)$ & $\mathrm{C} 125-\mathrm{C} 126-\mathrm{H} 126$ & 117.8 \\
\hline $\mathrm{N} 121-\mathrm{Cu}-\mathrm{N} 211$ & $108.8(2)$ & $\mathrm{C} 216-\mathrm{N} 211-\mathrm{C} 212$ & $120.1(7)$ \\
\hline $\mathrm{N} 221-\mathrm{Cu}-\mathrm{N} 121$ & $99.9(2)$ & $\mathrm{C} 216-\mathrm{N} 211-\mathrm{Cu}$ & $127.9(6)$ \\
\hline $\mathrm{N} 221-\mathrm{Cu}-\mathrm{N} 211$ & $80.5(2)$ & $\mathrm{C} 212-\mathrm{N} 211-\mathrm{Cu}$ & $111.7(5)$ \\
\hline $\mathrm{N} 111-\mathrm{Cu}-\mathrm{O} 31$ & $90.4(2)$ & $\mathrm{N} 211-\mathrm{C} 212-\mathrm{C} 213$ & $120.0(8)$ \\
\hline $\mathrm{N} 121-\mathrm{Cu}-\mathrm{O} 31$ & $150.0(2)$ & $\mathrm{N} 211-\mathrm{C} 212-\mathrm{C} 222$ & $115.2(7)$ \\
\hline
\end{tabular}




\begin{tabular}{|c|c|c|c|}
\hline $\mathrm{N} 211-\mathrm{Cu}-\mathrm{O} 31$ & $101.2(2)$ & $\mathrm{C} 213-\mathrm{C} 212-\mathrm{C} 222$ & $124.8(8)$ \\
\hline $\mathrm{N} 221-\mathrm{Cu}-\mathrm{O} 31$ & $87.2(2)$ & $\mathrm{C} 214-\mathrm{C} 213-\mathrm{C} 212$ & $120.3(9)$ \\
\hline $\mathrm{N} 111-\mathrm{Cu}-\mathrm{O} 32$ & $83.6(2)$ & $\mathrm{C} 214-\mathrm{C} 213-\mathrm{H} 213$ & 119.9 \\
\hline $\mathrm{N} 121-\mathrm{Cu}-\mathrm{O} 32$ & $96.7(2)$ & $\mathrm{C} 212-\mathrm{C} 213-\mathrm{H} 213$ & 119.9 \\
\hline $\mathrm{N} 211-\mathrm{Cu}-\mathrm{O} 32$ & $154.4(2)$ & $\mathrm{C} 213-\mathrm{C} 214-\mathrm{C} 215$ & $119.5(9)$ \\
\hline $\mathrm{N} 221-\mathrm{Cu}-\mathrm{O} 32$ & $92.6(2)$ & $\mathrm{C} 213-\mathrm{C} 214-\mathrm{H} 214$ & 120.3 \\
\hline $\mathrm{O} 31-\mathrm{Cu}-\mathrm{O} 32$ & $53.57(19)$ & $\mathrm{C} 215-\mathrm{C} 214-\mathrm{H} 214$ & 120.3 \\
\hline $\mathrm{C} 112-\mathrm{N} 111-\mathrm{C} 116$ & $118.9(6)$ & $\mathrm{C} 214-\mathrm{C} 215-\mathrm{C} 216$ & $119.2(9)$ \\
\hline $\mathrm{C} 112-\mathrm{N} 111-\mathrm{Cu}$ & $115.8(5)$ & $\mathrm{C} 214-\mathrm{C} 215-\mathrm{H} 215$ & 120.4 \\
\hline $\mathrm{C} 116-\mathrm{N} 111-\mathrm{Cu}$ & $124.6(6)$ & $\mathrm{C} 216-\mathrm{C} 215-\mathrm{H} 215$ & 120.4 \\
\hline $\mathrm{N} 111-\mathrm{C} 112-\mathrm{C} 113$ & $122.2(7)$ & $\mathrm{N} 211-\mathrm{C} 216-\mathrm{C} 215$ & $121.0(8)$ \\
\hline $\mathrm{N} 111-\mathrm{C} 112-\mathrm{C} 122$ & $114.2(7)$ & $\mathrm{N} 211-\mathrm{C} 216-\mathrm{H} 216$ & 119.5 \\
\hline $\mathrm{C} 113-\mathrm{C} 112-\mathrm{C} 122$ & $123.6(8)$ & $\mathrm{C} 215-\mathrm{C} 216-\mathrm{H} 216$ & 119.5 \\
\hline $\mathrm{C} 114-\mathrm{C} 113-\mathrm{C} 112$ & $117.7(8)$ & $\mathrm{C} 226-\mathrm{N} 221-\mathrm{C} 222$ & $120.1(6)$ \\
\hline $\mathrm{C} 114-\mathrm{C} 113-\mathrm{H} 113$ & 121.2 & $\mathrm{C} 226-\mathrm{N} 221-\mathrm{Cu}$ & $124.7(5)$ \\
\hline $\mathrm{C} 112-\mathrm{C} 113-\mathrm{H} 113$ & 121.2 & $\mathrm{C} 222-\mathrm{N} 221-\mathrm{Cu}$ & $114.5(4)$ \\
\hline $\mathrm{C} 113-\mathrm{C} 114-\mathrm{C} 115$ & $121.2(8)$ & $\mathrm{N} 221-\mathrm{C} 222-\mathrm{C} 223$ & $118.9(6)$ \\
\hline $\mathrm{C} 113-\mathrm{C} 114-\mathrm{H} 114$ & 119.4 & $\mathrm{~N} 221-\mathrm{C} 222-\mathrm{C} 212$ & $116.6(7)$ \\
\hline $\mathrm{C} 115-\mathrm{C} 114-\mathrm{H} 114$ & 119.4 & $\mathrm{C} 223-\mathrm{C} 222-\mathrm{C} 212$ & $124.4(7)$ \\
\hline $\mathrm{C} 114-\mathrm{C} 115-\mathrm{C} 116$ & $118.7(8)$ & $\mathrm{C} 224-\mathrm{C} 223-\mathrm{C} 222$ & $119.9(7)$ \\
\hline $\mathrm{C} 114-\mathrm{C} 115-\mathrm{H} 115$ & 120.7 & $\mathrm{C} 224-\mathrm{C} 223-\mathrm{H} 223$ & 120.0 \\
\hline $\mathrm{C} 116-\mathrm{C} 115-\mathrm{H} 115$ & 120.7 & $\mathrm{C} 222-\mathrm{C} 223-\mathrm{H} 223$ & 120.0 \\
\hline $\mathrm{N} 111-\mathrm{C} 116-\mathrm{C} 115$ & $121.1(8)$ & $\mathrm{C} 225-\mathrm{C} 224-\mathrm{C} 223$ & $119.1(7)$ \\
\hline $\mathrm{N} 111-\mathrm{C} 116-\mathrm{H} 116$ & 119.4 & $\mathrm{C} 225-\mathrm{C} 224-\mathrm{H} 224$ & 120.4 \\
\hline $\mathrm{C} 115-\mathrm{C} 116-\mathrm{H} 116$ & 119.4 & $\mathrm{C} 223-\mathrm{C} 224-\mathrm{H} 224$ & 120.4 \\
\hline $\mathrm{C} 126-\mathrm{N} 121-\mathrm{C} 122$ & $117.8(7)$ & $\mathrm{C} 226-\mathrm{C} 225-\mathrm{C} 224$ & $119.2(8)$ \\
\hline $\mathrm{C} 126-\mathrm{N} 121-\mathrm{Cu}$ & $127.6(5)$ & $\mathrm{C} 226-\mathrm{C} 225-\mathrm{H} 225$ & 120.4 \\
\hline $\mathrm{C} 122-\mathrm{N} 121-\mathrm{Cu}$ & $114.5(5)$ & $\mathrm{C} 224-\mathrm{C} 225-\mathrm{H} 225$ & 120.4 \\
\hline $\mathrm{N} 121-\mathrm{C} 122-\mathrm{C} 123$ & $121.7(7)$ & $\mathrm{N} 221-\mathrm{C} 226-\mathrm{C} 225$ & $122.6(7)$ \\
\hline $\mathrm{N} 121-\mathrm{C} 122-\mathrm{C} 112$ & $113.7(7)$ & $\mathrm{N} 221-\mathrm{C} 226-\mathrm{H} 226$ & 118.7 \\
\hline $\mathrm{C} 123-\mathrm{C} 122-\mathrm{C} 112$ & $124.5(8)$ & $\mathrm{C} 225-\mathrm{C} 226-\mathrm{H} 226$ & 118.7 \\
\hline $\mathrm{C} 124-\mathrm{C} 123-\mathrm{C} 122$ & $117.5(8)$ & $\mathrm{O} 33-\mathrm{N} 3-\mathrm{O} 31$ & $122.5(8)$ \\
\hline $\mathrm{C} 124-\mathrm{C} 123-\mathrm{H} 123$ & 121.3 & $\mathrm{O} 33-\mathrm{N} 3-\mathrm{O} 32$ & $120.2(9)$ \\
\hline $\mathrm{C} 122-\mathrm{C} 123-\mathrm{H} 123$ & 121.3 & $\mathrm{O} 31-\mathrm{N} 3-\mathrm{O} 32$ & $117.3(8)$ \\
\hline $\mathrm{C} 125-\mathrm{C} 124-\mathrm{C} 123$ & $122.4(9)$ & $\mathrm{N} 3-\mathrm{O} 31-\mathrm{Cu}$ & $104.1(5)$ \\
\hline $\mathrm{C} 125-\mathrm{C} 124-\mathrm{H} 124$ & 118.8 & $\mathrm{~N} 3-\mathrm{O} 32-\mathrm{Cu}$ & $85.0(5)$ \\
\hline $\mathrm{C} 123-\mathrm{C} 124-\mathrm{H} 124$ & 118.8 & $\mathrm{O} 43-\mathrm{N} 4-\mathrm{O} 41$ & $121.7(8)$ \\
\hline $\mathrm{C} 124-\mathrm{C} 125-\mathrm{C} 126$ & $116.0(8)$ & $\mathrm{O} 43-\mathrm{N} 4-\mathrm{O} 42$ & $120.4(9)$ \\
\hline $\mathrm{C} 124-\mathrm{C} 125-\mathrm{H} 125$ & 122.0 & $\mathrm{O} 41-\mathrm{N} 4-\mathrm{O} 42$ & $117.9(8)$ \\
\hline $\mathrm{C} 126-\mathrm{C} 125-\mathrm{H} 125$ & 122.0 & & \\
\hline $\mathrm{N} 121-\mathrm{Cu}-\mathrm{N} 111-\mathrm{C} 112$ & $-9.4(5)$ & $\mathrm{N} 121-\mathrm{Cu}-\mathrm{N} 211-\mathrm{C} 212$ & $97.2(5)$ \\
\hline $\mathrm{N} 211-\mathrm{Cu}-\mathrm{N} 111-\mathrm{C} 112$ & $-116.7(5)$ & $\mathrm{O} 31-\mathrm{Cu}-\mathrm{N} 211-\mathrm{C} 212$ & $-85.3(5)$ \\
\hline $\mathrm{O} 31-\mathrm{Cu}-\mathrm{N} 111-\mathrm{C} 112$ & $141.7(5)$ & $\mathrm{O} 32-\mathrm{Cu}-\mathrm{N} 211-\mathrm{C} 212$ & $-76.2(7)$ \\
\hline $\mathrm{O} 32-\mathrm{Cu}-\mathrm{N} 111-\mathrm{C} 112$ & $88.5(5)$ & $\mathrm{C} 216-\mathrm{N} 211-\mathrm{C} 212-\mathrm{C} 213$ & $1.7(11)$ \\
\hline $\mathrm{N} 121-\mathrm{Cu}-\mathrm{N} 111-\mathrm{C} 116$ & $-179.7(6)$ & $\mathrm{Cu}-\mathrm{N} 211-\mathrm{C} 212-\mathrm{C} 213$ & $-172.0(5)$ \\
\hline $\mathrm{N} 211-\mathrm{Cu}-\mathrm{N} 111-\mathrm{C} 116$ & $73.0(6)$ & $\mathrm{C} 216-\mathrm{N} 211-\mathrm{C} 212-\mathrm{C} 222$ & $-179.4(6)$ \\
\hline $\mathrm{O} 31-\mathrm{Cu}-\mathrm{N} 111-\mathrm{C} 116$ & $-28.5(6)$ & $\mathrm{Cu}-\mathrm{N} 211-\mathrm{C} 212-\mathrm{C} 222$ & $6.9(7)$ \\
\hline
\end{tabular}




\begin{tabular}{|c|c|}
\hline $\mathrm{O} 32-\mathrm{Cu}-\mathrm{N} 111-\mathrm{C} 116$ & $-81.8(6)$ \\
\hline $\mathrm{C} 116-\mathrm{N} 111-\mathrm{C} 112-\mathrm{C} 113$ & $1.2(11)$ \\
\hline $\mathrm{Cu}-\mathrm{N} 111-\mathrm{C} 112-\mathrm{C} 113$ & $-169.7(6)$ \\
\hline $\mathrm{C} 116-\mathrm{N} 111-\mathrm{C} 112-\mathrm{C} 122$ & $-177.3(6)$ \\
\hline $\mathrm{Cu}-\mathrm{N} 111-\mathrm{C} 112-\mathrm{C} 122$ & $11.9(8)$ \\
\hline $\mathrm{N} 111-\mathrm{C} 112-\mathrm{C} 113-\mathrm{C} 114$ & $-2.1(12)$ \\
\hline $\mathrm{C} 122-\mathrm{C} 112-\mathrm{C} 113-\mathrm{C} 114$ & $176.2(6)$ \\
\hline $\mathrm{C} 112-\mathrm{C} 113-\mathrm{C} 114-\mathrm{C} 115$ & $-0.5(12)$ \\
\hline $\mathrm{C} 113-\mathrm{C} 114-\mathrm{C} 115-\mathrm{C} 116$ & $3.9(14)$ \\
\hline $\mathrm{C} 112-\mathrm{N} 111-\mathrm{C} 116-\mathrm{C} 115$ & $2.5(12)$ \\
\hline $\mathrm{Cu}-\mathrm{N} 111-\mathrm{C} 116-\mathrm{C} 115$ & $172.4(7)$ \\
\hline $\mathrm{C} 114-\mathrm{C} 115-\mathrm{C} 116-\mathrm{N} 111$ & $-5.0(14)$ \\
\hline $\mathrm{N} 111-\mathrm{Cu}-\mathrm{N} 121-\mathrm{C} 126$ & $-171.7(6)$ \\
\hline $\mathrm{N} 221-\mathrm{Cu}-\mathrm{N} 121-\mathrm{C} 126$ & $12.1(6)$ \\
\hline $\mathrm{N} 211-\mathrm{Cu}-\mathrm{N} 121-\mathrm{C} 126$ & $-71.1(6)$ \\
\hline $\mathrm{O} 31-\mathrm{Cu}-\mathrm{N} 121-\mathrm{C} 126$ & $113.9(7)$ \\
\hline $\mathrm{O} 32-\mathrm{Cu}-\mathrm{N} 121-\mathrm{C} 126$ & $106.0(6)$ \\
\hline $\mathrm{N} 111-\mathrm{Cu}-\mathrm{N} 121-\mathrm{C} 122$ & $5.0(5)$ \\
\hline $\mathrm{N} 221-\mathrm{Cu}-\mathrm{N} 121-\mathrm{C} 122$ & $-171.2(5)$ \\
\hline $\mathrm{N} 211-\mathrm{Cu}-\mathrm{N} 121-\mathrm{C} 122$ & $105.5(5)$ \\
\hline $\mathrm{O} 31-\mathrm{Cu}-\mathrm{N} 121-\mathrm{C} 122$ & $-69.5(7)$ \\
\hline $\mathrm{O} 32-\mathrm{Cu}-\mathrm{N} 121-\mathrm{C} 122$ & $-77.4(5)$ \\
\hline $\mathrm{C} 126-\mathrm{N} 121-\mathrm{C} 122-\mathrm{C} 123$ & $0.7(10)$ \\
\hline $\mathrm{Cu}-\mathrm{N} 121-\mathrm{C} 122-\mathrm{C} 123$ & $-176.2(5)$ \\
\hline $\mathrm{C} 126-\mathrm{N} 121-\mathrm{C} 122-\mathrm{C} 112$ & $176.8(6)$ \\
\hline $\mathrm{Cu}-\mathrm{N} 121-\mathrm{C} 122-\mathrm{C} 112$ & $-0.2(7)$ \\
\hline $\mathrm{N} 111-\mathrm{C} 112-\mathrm{C} 122-\mathrm{N} 121$ & $-7.5(9)$ \\
\hline $\mathrm{C} 113-\mathrm{C} 112-\mathrm{C} 122-\mathrm{N} 121$ & $174.0(7)$ \\
\hline $\mathrm{N} 111-\mathrm{C} 112-\mathrm{C} 122-\mathrm{C} 123$ & $168.4(7)$ \\
\hline $\mathrm{C} 113-\mathrm{C} 112-\mathrm{C} 122-\mathrm{C} 123$ & $-10.1(11)$ \\
\hline $\mathrm{N} 121-\mathrm{C} 122-\mathrm{C} 123-\mathrm{C} 124$ & $1.3(10)$ \\
\hline $\mathrm{C} 112-\mathrm{C} 122-\mathrm{C} 123-\mathrm{C} 124$ & $-174.3(7)$ \\
\hline $\mathrm{C} 122-\mathrm{C} 123-\mathrm{C} 124-\mathrm{C} 125$ & $-1.7(12)$ \\
\hline $\mathrm{C} 123-\mathrm{C} 124-\mathrm{C} 125-\mathrm{C} 126$ & $0.1(12)$ \\
\hline $\mathrm{C} 122-\mathrm{N} 121-\mathrm{C} 126-\mathrm{C} 125$ & $-2.5(10)$ \\
\hline $\mathrm{Cu}-\mathrm{N} 121-\mathrm{C} 126-\mathrm{C} 125$ & $174.0(5)$ \\
\hline $\mathrm{C} 124-\mathrm{C} 125-\mathrm{C} 126-\mathrm{N} 121$ & $2.1(12)$ \\
\hline $\mathrm{N} 111-\mathrm{Cu}-\mathrm{N} 211-\mathrm{C} 216$ & $8.6(7)$ \\
\hline $\mathrm{N} 221-\mathrm{Cu}-\mathrm{N} 211-\mathrm{C} 216$ & $-173.3(6)$ \\
\hline $\mathrm{N} 121-\mathrm{Cu}-\mathrm{N} 211-\mathrm{C} 216$ & $-75.9(6)$ \\
\hline $\mathrm{O} 31-\mathrm{Cu}-\mathrm{N} 211-\mathrm{C} 216$ & $101.5(6)$ \\
\hline $\mathrm{O} 32-\mathrm{Cu}-\mathrm{N} 211-\mathrm{C} 216$ & $110.7(7)$ \\
\hline $\mathrm{N} 111-\mathrm{Cu}-\mathrm{N} 211-\mathrm{C} 212$ & $-178.3(5)$ \\
\hline $\mathrm{N} 221-\mathrm{Cu}-\mathrm{N} 211-\mathrm{C} 212$ & $-0.1(5)$ \\
\hline
\end{tabular}

\begin{tabular}{|c|c|}
\hline $\mathrm{N} 211-\mathrm{C} 212-\mathrm{C} 213-\mathrm{C} 214$ & $-0.3(12)$ \\
\hline $\mathrm{C} 222-\mathrm{C} 212-\mathrm{C} 213-\mathrm{C} 214$ & $-179.1(7)$ \\
\hline $\mathrm{C} 212-\mathrm{C} 213-\mathrm{C} 214-\mathrm{C} 215$ & $-1.1(14)$ \\
\hline $\mathrm{C} 213-\mathrm{C} 214-\mathrm{C} 215-\mathrm{C} 216$ & $1.0(15)$ \\
\hline $\mathrm{C} 212-\mathrm{N} 211-\mathrm{C} 216-\mathrm{C} 215$ & $-1.8(11)$ \\
\hline $\mathrm{Cu}-\mathrm{N} 211-\mathrm{C} 216-\mathrm{C} 215$ & $170.8(6)$ \\
\hline $\mathrm{C} 214-\mathrm{C} 215-\mathrm{C} 216-\mathrm{N} 211$ & $0.4(13)$ \\
\hline $\mathrm{N} 121-\mathrm{Cu}-\mathrm{N} 221-\mathrm{C} 226$ & $74.6(6)$ \\
\hline $\mathrm{N} 211-\mathrm{Cu}-\mathrm{N} 221-\mathrm{C} 226$ & $-177.8(6$ \\
\hline $\mathrm{O} 31-\mathrm{Cu}-\mathrm{N} 221-\mathrm{C} 226$ & $-76.0(6)$ \\
\hline $\mathrm{O} 32-\mathrm{Cu}-\mathrm{N} 221-\mathrm{C} 226$ & $-22.7(6)$ \\
\hline $\mathrm{N} 121-\mathrm{Cu}-\mathrm{N} 221-\mathrm{C} 222$ & $-114.7(5)$ \\
\hline $\mathrm{N} 211-\mathrm{Cu}-\mathrm{N} 221-\mathrm{C} 222$ & $-7.1(5)$ \\
\hline $\mathrm{O} 31-\mathrm{Cu}-\mathrm{N} 221-\mathrm{C} 222$ & $94.7(5)$ \\
\hline $\mathrm{O} 32-\mathrm{Cu}-\mathrm{N} 221-\mathrm{C} 222$ & $148.0(5)$ \\
\hline $\mathrm{C} 226-\mathrm{N} 221-\mathrm{C} 222-\mathrm{C} 223$ & $4.5(10)$ \\
\hline $\mathrm{Cu}-\mathrm{N} 221-\mathrm{C} 222-\mathrm{C} 223$ & $-166.7(5$ \\
\hline $\mathrm{C} 226-\mathrm{N} 221-\mathrm{C} 222-\mathrm{C} 212$ & $-175.7(6)$ \\
\hline $\mathrm{Cu}-\mathrm{N} 221-\mathrm{C} 222-\mathrm{C} 212$ & $13.1(8)$ \\
\hline $\mathrm{N} 211-\mathrm{C} 212-\mathrm{C} 222-\mathrm{N} 221$ & $-13.4(9)$ \\
\hline $\mathrm{C} 213-\mathrm{C} 212-\mathrm{C} 222-\mathrm{N} 221$ & $165.5(7)$ \\
\hline $\mathrm{N} 211-\mathrm{C} 212-\mathrm{C} 222-\mathrm{C} 223$ & $166.4(6)$ \\
\hline $\mathrm{C} 213-\mathrm{C} 212-\mathrm{C} 222-\mathrm{C} 223$ & $-14.7(11)$ \\
\hline $\mathrm{N} 221-\mathrm{C} 222-\mathrm{C} 223-\mathrm{C} 224$ & $-1.9(10)$ \\
\hline $\mathrm{C} 212-\mathrm{C} 222-\mathrm{C} 223-\mathrm{C} 224$ & $178.3(6)$ \\
\hline $\mathrm{C} 222-\mathrm{C} 223-\mathrm{C} 224-\mathrm{C} 225$ & $-1.4(11)$ \\
\hline $\mathrm{C} 223-\mathrm{C} 224-\mathrm{C} 225-\mathrm{C} 226$ & $2.1(12)$ \\
\hline $\mathrm{C} 222-\mathrm{N} 221-\mathrm{C} 226-\mathrm{C} 225$ & $-3.9(11)$ \\
\hline $\mathrm{Cu}-\mathrm{N} 221-\mathrm{C} 226-\mathrm{C} 225$ & $166.3(6)$ \\
\hline $\mathrm{C} 224-\mathrm{C} 225-\mathrm{C} 226-\mathrm{N} 221$ & $0.5(12)$ \\
\hline $\mathrm{O} 33-\mathrm{N} 3-\mathrm{O} 31-\mathrm{Cu}$ & $179.6(7)$ \\
\hline $\mathrm{O} 32-\mathrm{N} 3-\mathrm{O} 31-\mathrm{Cu}$ & $-2.2(8)$ \\
\hline $\mathrm{N} 111-\mathrm{Cu}-\mathrm{O} 31-\mathrm{N} 3$ & $-80.5(5)$ \\
\hline $\mathrm{N} 221-\mathrm{Cu}-\mathrm{O} 31-\mathrm{N} 3$ & $96.5(5)$ \\
\hline $\mathrm{N} 121-\mathrm{Cu}-\mathrm{O} 31-\mathrm{N} 3$ & $-8.5(8)$ \\
\hline $\mathrm{N} 211-\mathrm{Cu}-\mathrm{O} 31-\mathrm{N} 3$ & $176.3(5)$ \\
\hline $\mathrm{O} 32-\mathrm{Cu}-\mathrm{O} 31-\mathrm{N} 3$ & $1.2(4)$ \\
\hline $\mathrm{O} 33-\mathrm{N} 3-\mathrm{O} 32-\mathrm{Cu}$ & $-180.0(8$ \\
\hline $\mathrm{O} 31-\mathrm{N} 3-\mathrm{O} 32-\mathrm{Cu}$ & $1.8(7)$ \\
\hline $\mathrm{N} 111-\mathrm{Cu}-\mathrm{O} 32-\mathrm{N} 3$ & $94.1(5)$ \\
\hline $\mathrm{N} 221-\mathrm{Cu}-\mathrm{O} 32-\mathrm{N} 3$ & $-85.7(5)$ \\
\hline $\mathrm{N} 121-\mathrm{Cu}-\mathrm{O} 32-\mathrm{N} 3$ & $174.0(5)$ \\
\hline $\mathrm{N} 211-\mathrm{Cu}-\mathrm{O} 32-\mathrm{N} 3$ & $-12.3(8)$ \\
\hline $\mathrm{O} 31-\mathrm{Cu}-\mathrm{O} 32-\mathrm{N} 3$ & $-1.1(4)$ \\
\hline
\end{tabular}

